\title{
O campo de atenção à saúde de sujeitos com problemáticas decorrentes do uso de álcool: apontamentos para a formulação de práticas de cuidado
}

\section{The field of health attention to individuals with problems in consequence of the alcohol use: notes for the formulation of health care practices}

Ana Lucia Marinho Marques', Elisabete Ferreira Mângia²

\begin{abstract}
MARQUES, A. L. M.; MANGIA, E. F. O campo de atenção à saúde de sujeitos com problemáticas decorrentes do uso de álcool: apontamentos para a formulação de práticas de cuidado. Rev. Ter. Ocup. Univ. São Paulo, v. 20, n. 1, p. 43-48, jan./abr. 2009.

RESUMO: Os problemas decorrentes do uso nocivo ou prejudicial de álcool trazem impactos relevantes para o campo da saúde pública e requerem o desenvolvimento de políticas de atenção e cuidado. Neste cenário, ganha destaque a implementação de uma rede de atenção integral, composta por ações e serviços de diversos níveis assistenciais e que busquem responder às complexas necessidades dos sujeitos atendidos. Dessa rede, destacamos o papel dos Centros de Atenção Psicossocial para Atendimento de Pacientes com Transtornos Causados pelo Uso Prejudicial e/ou Dependência de Álcool e outras Drogas - CAPSad. Situados no território onde vivem os sujeitos atendidos, tais serviços podem ser espaços privilegiados na articulação de ações e contextos e na construção de projetos terapêuticos singulares, comprometidos com o princípio de atenção integral e com o complexo mundo de necessidades apresentadas pelos usuários.
\end{abstract}

DESCRITORES: Transtornos relacionados ao uso de substâncias. Alcoolismo. Políticas públicas de saúde. Serviços comunitários de saúde mental. Terapia ocupacional/tendências.

\footnotetext{
1. Terapeuta Ocupacional, Mestranda do Programa de Pós-Graduação em Ciências da Reabilitação da FMUSP.

2. Profa. Dra. do Departamento de Fisioterapia, Fonoaudiologia e Terapia Ocupacional da FMUSP.

Endereço para correspondência: Departamento de Fisioterapia, Fonoaudiologia e Terapia Ocupacional da FMUSP. Rua Cipotânea, 51, Cidade Universitária, São Paulo, SP, CEP: 05360-160.
} 


\section{INTRODUÇÃO}

$\mathrm{A}$ s questões decorrentes do uso das substâncias psicoativas se colocam como problema global, que mobiliza diversos setores sociais. Nas sociedades contemporâneas, este tema assumiu lugar central no debate público, principalmente por sua representação enquanto ameaça para a saúde pessoal e coletiva e por sua associação com a criminalidade e a violência urbana (SIMÕES, 2008).

No campo da saúde pública, observa-se o aumento da preocupação sobre o impacto do uso nocivo ou prejudicial de álcool e outras substâncias psicoativas sobre a saúde dos indivíduos e das populações. Como resultado, tem-se construído alternativas de atenção a saúde que considerem os sujeitos em seus particulares contextos de vida, e os diferenciados tipos de cuidado de que necessitam.

Neste artigo, buscaremos contextualizar e problematizar esta questão e apresentar possibilidades de desenvolvimento de estratégias de cuidado de acordo com a literatura atualizada.

\section{Impacto do uso do álcool e outras substâncias psicoativas para o campo da saúde pública}

Em todo o mundo, são significativas as diversas problemáticas decorrentes de transtornos mentais, tanto por atingirem porcentagem considerável da população, quanto pelos impactos e sofrimento que causam na vida dos indivíduos. Dentro deste quadro, estão os transtornos mentais e comportamentais resultantes do uso de substâncias psicoativas. Estes incluem: transtornos devidos ao uso de álcool, opiáceos como ópio ou heroína, canabinóides como maconha, sedativos e hipnóticos, cocaína e outros estimulantes, alucinógenos, fumo e solventes voláteis. A prevalência do uso de substâncias psicoativas, e de transtornos e risco de infecções decorrentes do mesmo variam entre regiões e populações (OMS/OPAS, 2001; WHO, 2004).

As substâncias psicoativas ou drogas psicoativas podem ser definidas como "aquelas que modificam o estado de consciência do usuário" (SEIBEL; TOSCANO, 2001), afetando processos mentais tais como cognição e humor (BRASIL, 2006a). Utilizadas tanto em situações de celebração como de sofrimento, quase todas as drogas fazem parte de ritos de sociabilidade, cura, devoção, consolo e prazer (CARNEIRO, 2005).

O álcool é uma substância psicoativa cujo uso é tolerado nas sociedades ocidentais e até incentivado nas campanhas publicitárias que o associam à juventude, à beleza, ao sucesso, vinculado à imagem de figuras públicas. Faz parte de situações de lazer, recreação e descontração e é a substância mais utilizada em momentos de festas e comemorações, sendo nessas situações em que os sujeitos, geralmente, experimentam essa substância pela primeira vez, junto com familiares e amigos (CARNEIRO, 2005; ACSELRAD, 2005).

No entanto, diversas pesquisas apontam o álcool e o tabaco como substâncias que têm as mais graves conseqüências para a saúde pública, e que podem acarretar diversos danos nas esferas sociais e individuais. De acordo com dados da Organização Mundial da Saúde, estima-se que cerca de 2 bilhões de pessoas em todo o mundo consomem bebidas alcoólicas - o que corresponde a aproximadamente $40 \%$ da população mundial acima de 15 anos - e cerca de 76.3 milhões apresentam problemáticas decorrentes do uso dessa substância(WHO, 2002, 2004).

Em muitas partes do mundo, é considerável a carga global relacionada ao consumo de álcool, tanto em termos de morbidade quanto de mortalidade: pode trazer conseqüências diversas e está relacionado a mais de 60 tipos de doenças ou lesões, além de episódios de violência, homicídios e acidentes; está associado a 3,2\% de todas as mortes no mundo (sendo, aproximadamente, $6 \%$ de todas as mortes entre homens e $1 \%$ entre as mulheres) e $4 \%$ do total de anos de vida comprometidos por alguma incapacidade (WHO, 2002, 2004).

Pesquisas consideram que $12 \%$ da população brasileira apresentam problemas decorrentes do uso de álcool, associados ao uso nocivo e à dependência. Este índice é especialmente significativo em termos de saúde pública, pois uma parte substancial dessas pessoas necessita de alguma forma de tratamento ou apresenta algum quadro clínico que requer ações do sistema de saúde (CARLINI et al., 2006; LARANJEIRA et al., 2007).

$\mathrm{Na}$ Tabela 1, encontramos a freqüência de internações por transtornos devido ao uso de álcool e outras substâncias psicoativas, por faixa etária, entre janeiro e março de 2006. Mais de $70 \%$ das internações pelo uso prejudicial de substâncias psicoativas, em Hospitais Psiquiátricos e Hospitais Gerais, estão associadas ao uso do álcool. Na população dos hospitais psiquiátricos brasileiros, quase um quarto dos pacientes foram internados por transtornos ligados ao consumo do álcool, e em torno de $40 \%$ apresentam o consumo prejudicial de álcool como parte do quadro clínico (BRASIL, 2003, 2006b).

No campo de atenção aos sujeitos com transtornos decorrentes do uso de substâncias psicoativas, o uso prejudicial de álcool é considerado o principal ponto a ser problematizado. Por muito tempo, o Estado não assumiu 
MARQUES, A. L. M.; MANGIA, E. F. O campo de atenção à saúde. Rev. Ter. Ocup. Univ. São Paulo, v. 20, n. 1, p. 43-48, jan./abr. 2009.

sua responsabilidade e as políticas públicas direcionadas a questão do consumo prejudicial do álcool, bem como de outras drogas, mostravam, até recentemente, uma lacuna de iniciativas consistentes e regulares (DELGADO, 2005).

TABELA 1. Internações por faixa etária segundo lista de morbidade CID10: Transtornos Mentais e comportamentais devido ao uso de álcool e Transtornos Mentais e comportamentais devido ao uso de outras substâncias psicoativas - janeiro a março de 2006

\begin{tabular}{llll}
\hline Faixa etária & Álcool & Outras substâncias & Total \\
\hline 1 a 9 & 10 & 133 & 143 \\
10 a 14 & 23 & 92 & 115 \\
15 a 19 & 157 & 846 & 1003 \\
20 a 24 & 576 & 1634 & 2210 \\
25 a 29 & 1360 & 1416 & 2776 \\
30 a 34 & 2287 & 988 & 3275 \\
35 a 39 & 3205 & 659 & 3864 \\
40 a 44 & 3686 & 426 & 4112 \\
45 a 49 & 3171 & 281 & 3452 \\
50 a 54 & 2176 & 156 & 2332 \\
55 a 59 & 1216 & 56 & 1272 \\
60 a 64 & 635 & 40 & 675 \\
65 a 69 & 347 & 14 & 361 \\
70 a 74 & 163 & 9 & 172 \\
75 a 79 & 58 & 9 & 67 \\
$>80$ & 25 & 8 & 33 \\
\hline Total & 19095 & 6767 & 25862 \\
$\%$ & 73,83 & 26,17 & 100,00 \\
\hline
\end{tabular}

Fonte: Saúde Mental em dados nº 2, Ministério da Saúde, 2006.

A ausência de uma política pública de saúde consistente produziu o deslocamento deste problema para instituições da justiça, segurança pública, pedagogia, associações filantrópicas e religiosas. Como resultado, observou-se a criação e disseminação de práticas e modelos de cuidado disciplinares ou de cunho religioso baseados predominantemente na internação e segregação e tendo como principal meta a abstinência (BRASIL, 2003, 2007a).

Além disso, a classificação das drogas em lícitas e ilícitas produziu o efeito de concentrar a preocupação do governo e da sociedade na repressão ao consumo das substâncias consideradas ilegais, reservando lugar secundário para o álcool e o tabaco, justamente aquelas que têm sido consideradas as mais prejudiciais à saúde da população (DELGADO, 2005).

Nos últimos anos, o Ministério da Saúde passou a formular diretrizes e propostas de atenção para abordar essa problemática. Em 2002, a Portaria GM 816 instituiu, no âmbito do Sistema Único de Saúde, o Programa Nacional de Atenção Comunitária Integrada a Usuários de Álcool e Outras Drogas, com o objetivo de organizar demandas e articular ações de assistência e prevenção desenvolvidas nas esferas de governo federal, estadual e municipal (BRASIL, 2002).

Em 2003, foi criado o Grupo de Trabalho em Álcool e Outras Drogas (GAOD) composto por representantes de diversas áreas, com o intuito de se discutir de forma ampla essa questão. Como resultado, foi apresentado o documento: A Política do Ministério da Saúde para Atenção Integral a Usuários de álcool e outras drogas. Esse documento contempla diretrizes para a abordagem e apresenta a avaliação, baseada em dados epidemiológicos, que considera que o uso de álcool e as problemáticas dele advindas deve ser entendido e trabalhado como questão de saúde pública (BRASIL, 2003).

Aponta também para a necessidade de incluir os usuários/dependentes de álcool e outras drogas em uma rede ampliada de cuidados; de modificar a legislação vigente sobre álcool e outras drogas aos objetivos da saúde pública; e de adotar a estratégia de redução de danos como alternativa eficaz no tratamento e na prevenção (BRASIL, 2003, 2007a).

Tal perspectiva reconhece a necessidade de se abordar os danos decorrentes do abuso/uso prejudicial de substâncias psicoativas em um contexto amplo, considerando suas 
implicações sociais, psicológicas, econômicas e políticas na construção de propostas de atenção (BRASIL, 2003).

A discussão e a construção de ações devem ter como diretriz a busca da superação da tendência de criminalização do consumo de drogas, enfatizar o respeito aos direitos humanos e construir alternativas de saúde pública eficazes e acessíveis aos sujeitos que necessitam de cuidados (BRASIL, 2009a).

\section{A assistência comunitária e integral no campo dos transtornos decorrentes do uso de álcool e outras drogas}

As recomendações propostas pelo Ministério da Saúde são aceitas internacionalmente e se situam no contexto das propostas de construção de políticas e serviços de saúde baseados na comunidade, que partem da crítica à hospitalização e à abordagem focalizada na doença.

A atenção centrada na comunidade baseia-se na proposta de atender aos sujeitos em seu território a partir de um enfoque global, contemplando as suas múltiplas necessidades. Nessa perspectiva, assumir o atendimento na comunidade implica na articulação de uma rede de serviços, próximos a residência das pessoas, acessíveis e centrados nas necessidades dos sujeitos atendidos. As ações a serem realizadas devem buscar atender ao individuo integralmente e consideram a importância da continuidade da atenção (OMS, 2001).

Um serviço de saúde mental baseado na comunidade é aquele que se dispõe a oferecer cuidado efetivo para uma população definida, considerando suas vulnerabilidades, e que trabalha em colaboração com outros serviços e agências locais. A partir de uma abordagem combinada com o campo da saúde pública, esse cuidado deve estar voltado para a saúde dos indivíduos e das populações, considerando as relações existentes entre contextos sociais e transtornos mentais. Ademais, deve considerar prevenção, tratamento e reabilitação como um continuum, e não estágios separados (THORNICROFT; TANSELLA, 2008).

É nesse contexto que se inscrevem as propostas para o campo de atenção e cuidados aos sujeitos com problemáticas decorrentes do uso de álcool e outras drogas. Internacionalmente, tem-se preconizado o acompanhamento integral e contínuo, articulando diversas estratégias de intervenção de acordo com a lógica de atenção baseada na comunidade. A atenção comunitária, compartilhada, contínua e de longa duração possibilita identificar fatores de risco e de proteção para as recaídas, abordar as múltiplas problemáticas envolvidas, bem como acionar parcerias e recursos da comunidade que possam ser utilizados para estimular mudanças e reorganizar a vida cotidiana (OMS, 2001; UNITED NATIONS, 2003).

A OMS também privilegia a articulação de ações de intervenção breve nos contextos da atenção primária, objetivando a detecção precoce e a prevenção de danos à saúde dos indivíduos e das populações, bem como procurar intervir em casos que ainda não se configurem como síndrome de dependência (OMS, 2001).

A construção de uma rede articulada de serviços de saúde, que possa aumentar o acesso e o acolhimento dos usuários, ampla e flexível para prestar um cuidado integral e atender as distintas necessidades dos indivíduos é fundamental nesse cenário. Esta rede deve estar articulada e estabelecer parcerias com serviços de outros setores tais como educação, trabalho, promoção social (BRASIL, 2003; WHO, 2004).

A política de saúde mental brasileira está de acordo com essas diretrizes e passou a implementar serviços comunitários de saúde mental, de forma que o Programa de Atenção Integral a Usuários de Álcool e outras Drogas contemple ações: na atenção básica; atenção em CAPSad, ambulatórios e outras unidades extra-hospitalares especializadas; na atenção hospitalar de referência; e na rede de suporte social (associações de ajuda mútua e entidades da sociedade civil), complementar aos serviços disponibilizados pelo SUS (BRASIL, 2003, 2004c).

\section{Os CAPSad}

Os Centros de Atenção Psicossocial para Atendimento de Pacientes com Transtornos Causados pelo Uso Prejudicial e/ou Dependência de Álcool e outras Drogas CAPSad, instituídos a partir da Portaria n ${ }^{\circ} 336 / \mathrm{GM}$ (Brasil, 2002; Brasil, 2003) são considerados o ponto central e estratégico da nova rede de cuidados. Dados apresentados em outubro de 2008 indicam a existência de 182 CAPSad credenciados no MS, sendo 45 no estado de São Paulo (BRASIL, 2008).

Recomenda-se que os CAPSad ofereçam atenção diária a indivíduos que fazem uso prejudicial de álcool e outras drogas, com atendimentos individuais ou em grupo, oficinas terapêuticas, visitas domiciliares e condições para o repouso, bem como para a desintoxicação ambulatorial, daqueles que necessitem desse tipo de cuidados e que não demandem por atenção clínica hospitalar (BRASIL, 2004b).

Devem obedecer à lógica de oferta de cuidados integrais, com práticas terapêuticas, preventivas, 
de promoção de saúde, educativas e de reabilitação psicossocial devem, também, estar articulados à rede de atenção integral aos usuários de álcool e outras drogas; à rede de cuidados em saúde mental e à rede de cuidados em DST/AIDS. Espera-se, ainda, a adoção da estratégia de redução de danos para a qual são previstos incentivos do Ministério da Saúde (BRASIL, 2004c; BRASIL, 2005, BRASIL, 2009).

As ações de redução de danos são compreendidas como "intervenções de saúde pública que visam prevenir as consequiências negativas do uso de álcool e outras drogas" (BRASIL, 2005). Podem contemplar: a ampliação da rede de atenção e do acesso aos serviços de saúde; a distribuição de insumos (seringas, agulhas, cachimbos) aos usuários de drogas, com o objetivo de prevenir a infecção dos vírus HIV e Hepatites B e C; além de medidas educativas, de apoio e orientação (BRASIL, 2005).

Os CAPSad podem se constituir como espaços privilegiados na construção de projetos terapêuticos ao desenvolver estratégias que tenham como território de investimentos a vida concreta dos sujeitos e ao priorizar o direcionamento das práticas para os contextos reais de vida das pessoas (SARACENO, 1999; BRASIL, 2003; MÂNGIA; MURAMOTO, 2006).

\section{Considerações finais}

O debate sobre a atenção à saúde dos sujeitos com problemáticas decorrentes do uso de álcool, bem como de outras substâncias psicoativas, tem adquirido destaque e há, por parte de gestores, profissionais e pesquisadores, o reconhecimento da importância da formulação de políticas e o crescente investimento na construção de tecnologias de cuidado nesse campo.

$\mathrm{Na}$ implementação de uma rede integral e articulada de serviços e ações de cuidado, os CAPSad. assumem lugar estratégico. Podem se configurar como espaços privilegiados para a elaboração e articulação das diversas ações de saúde demandadas no processo de construção de linhas de cuidado dos sujeitos que buscam e necessitam de atendimento. $\mathrm{O}$ acompanhamento contínuo e cotidiano favorece a construção de processos de inclusão e trocas de bens sociais, recursos e afetos. Possibilita que os sujeitos ressignifiquem suas histórias, restaurem laços afetados ou rompidos, e promovam percursos que possibilitem a entrada e o pertencimento em espaços capazes de potencializar a produção de saúde e de vida (SARACENO, 1999; BRASIL, 2003).

Para tanto, o processo de construção de projetos terapêuticos nesses serviços deve ser centrado nas reais necessidades, desejos e potencialidades dos sujeitos e na co-responsabilização entre usuários, técnicos e outros atores envolvidos. Exige criatividade e flexibilidade na promoção de respostas aos sujeitos, considerando suas distintas trajetórias e contextos sócio-familiares, formas de consumo de substâncias psicoativas e tentativas de interrupção (SARACENO, 1999; BRASIL, 2003; MÂNGIA; MURAMOTO, 2006).

MARQUES, A. L. M.; MANGIA, E. F. The field of health attention to individuals with problems in consequence of the alcohol use: notes for the formulation of health care practices. Rev. Ter. Ocup. Univ. São Paulo, v. 20, n. 1, p. 43-48, jan./abr. 2009.

ABSTRACT: The problems caused by the harmful use of alcohol bring significant impacts to the public health field and require the development of attention and health care policies. In this scenario, the implementation of an integral care network is emphasized, consisting of actions and services of all assistance levels which respond to the complex needs of individuals. In this network, we focus on the role of Psychosocial Care Centers to patients with disorders in consequence of the harmful use and/or alcohol or other drugs dependence - CAPSad. Located on the territory where the attended individuals live, such services may be privileged places to the articulation of actions and contexts and to the construction of singular therapeutic projects, committed to the principle of integral care and to the complex world of users needs.

KEY WORDS: Substance-related disorders. Alcoholism. Community mental health services. Health public policy. Occupational therapy/trends. 


\section{REFERÊNCIAS}

ACSELRAD, G. A educação para a autonomia: construindo um discurso democrático sobre as drogas. In: ACSELRAD, G. (Org.). Avessos do prazer: drogas, aids e direitos humanos. 2. ed. Rio de Janeiro: Fiocruz, 2005. p. 183-212.

BRASIL. Ministério da Saúde, Secretaria de Atenção à Saúde, Departamento de Ações Programáticas Estratégicas, Coordenação de saúde mental. Saúde Mental em Dados, v. 1, n.1, n. 2, jan-jul, 2006b.

BRASIL. Ministério da Saúde, Secretaria de Atenção à Saúde, Departamento de Ações Programáticas Estratégicas, Coordenação Geral de Saúde Mental, Álcool e outras drogas. Saúde Mental Dados, v. 3, n. 5, out. 2008.

BRASIL. Ministério da Saúde. A política do Ministério da Saúde para a atenção integral a usuários de álcool e outras drogas. Brasília, 2003. (Série B. Textos Básicos de Saúde).

BRASIL. Ministério da Saúde. Legislação em Saúde Mental, 19902002. 4. ed. Brasília, 2002. (Série E. Legislação de Saúde).

BRASIL. Ministério da Saúde. Portaria n. 1059/GM, 04 de julho de 2005. Destina incentivo financeiro para o fomento de ações de redução de danos em Centros de Atenção Psicossocial para o Álcool e outras Drogas - CAPSad - e dá outras providências. Diário Oficial da União, Brasília, 2005.

BRASIL. Ministério da Saúde. Portaria n.2197, 04 de outubro de 2004. Redefine e amplia a atenção integral para usuários de álcool e outras drogas, no âmbito do Sistema Único de Saúde - SUS, e dá outras providências. Diário Oficial da União, Brasília, 2004c.

BRASIL. Ministério da Saúde. Saúde Mental no SUS: os Centros de Atenção Psicossocial. Brasília, 2004b. (Série F. Comunicação e Educação em Saúde).

BRASIL. Ministério da Saúde. Secretaria de Atenção à Saúde. Saúde mental no SUS: acesso, eqüidade, qualidade. Desafios para consolidar a mudança do modelo. Relatório de gestão 2008. Coordenação Nacional de Saúde Mental, Álcool \& Outras Drogas. Brasília, 2009a (fechado em 09/01/09).

BRASIL. Ministério da Saúde. Secretaria de Atenção à Saúde/ DAPE. Saúde mental no SUS: acesso ao tratamento e mudança do modelo de atenção. Relatório de Gestão 2003-2006. Brasília, 2007a.

BRASIL. Presidência da República. Gabinete de Segurança Institucional. Secretaria Nacional Antidrogas. Glossário de Álcool e Drogas. Tradução e notas: Bertolote, J.M. Brasília: Secretaria Nacional Antidrogas, 2006a.

CARLINI, E. A. et al. II Levantamento domiciliar sobre o uso de drogas psicotrópicas no Brasil: estudo envolvendo as 108 maiores cidades do país. São Paulo: Centro Brasileiro de Informações sobre Drogas Psicotrópicas (CEBRID), Universidade Federal de São Paulo (UNIFESP), 2006.

CARNEIRO, H. Pequena enciclopédia da história das drogas e bebidas. São Paulo: Campus, 2005.

DELGADO, P. G. Drogas: o desafio da saúde pública. In: ACSELRAD, G. (Org). Avessos do prazer: drogas, AIDS e direitos humanos. 2. ed. Rio de Janeiro: Fiocruz, 2005. p. 165-181.

LARANJEIRA, R.; PINSKY, I.; ZALESKY, M.; CAETANO, R. I Levantamento nacional sobre padrões do consumo de álcool na população brasileira. Brasília: Secretaria Nacional Antidrogas, 2007.

MÂNGIA, E. F.; MURAMOTO, M. Integralidade e construção de novas profissionalidades no contexto dos serviços substitutivos de saúde mental. Rev. Ter. Ocup. USP, v. 17, n. 3, p. 115-122, 2006.

ORGANIZAÇÃO MUNDIAL DA SAÚDE (OMS), Organização Pan-americana da Saúde (OPAS). Relatório sobre a saúde no mundo - 2001. Saúde mental: nova concepção, nova esperança. Organização Mundial da Saúde, 2001.

SARACENO, B. Libertando identidades: da reabilitação psicossocial à cidadania possível. Rio de Janeiro: Te cora, 1999.

SEIBEL, S. D.; TOSCANO JUNIOR, A. Conceitos básicos e classificação geral das substâncias psicoativas. In: SEIBEL, S. D.; TOSCANO JUNIOR, A. Dependência de drogas. São Paulo: Atheneu, 2001.

SIMÕES, J. A. Prefácio. In: LABATE, B. C. et al. (Orgs.). Drogas e cultura: novas perspectivas. Salvador: EDUFBA, 2008.

THORNICROFT, G; TANSELLA, M. Better Mental Health Care. Londres: Cambridge University Press, 2008.

UNITED NATIONS. International Drug Control Programme Vienna. Investing in drug abuse treatment: a discussion paper for policy makers. New York: United Nations, 2003.

WORLD HEALTH ORGANIZATION (WHO). Disease control priorities related to mental, neurological, developmental and substance abuse disorders. Mental health: evidence and research. Geneva: Department of Mental Health and Substance Abuse, 2004.

WORLD HEALTH ORGANIZATION (WHO). The World Health Report 2002 - Reducing risks, promoting healthy life. Geneva, 2002. 